\title{
Cardiopulmonary exercise testing and SPECT myocardial perfusion imaging: Pre-test probability is the key
}

\author{
Anthony A. Holmes, $M D{ }^{a}$ and Lawrence $M$. Phillips, $M^{a}$ \\ a Leon H. Charney Division of Cardiology, New York University Langone Medical Center, \\ New York, NY
}

Received May 29, 2017; accepted May 30, 2017

doi: 10.1007/s12350-017-0996-7

\section{See related article, pp. 92-106}

Three and a half million exercise stress tests with myocardial perfusion imaging (exercise MPI) were ordered in 2010 in the United States for patients without a history of coronary artery disease (CAD). ${ }^{1}$ This represents nearly double the number of exercise MPI tests ordered in the year 2000. ${ }^{1}$ Exercise MPI is an excellent diagnostic tool to detect flow-limiting CAD, but it is limited in its ability to detect alternative non-cardiac etiologies of symptoms for which patients are often referred for exercise MPI, including reduced exercise tolerance and dyspnea. ${ }^{2}$ In the present era of evaluating cost effectiveness of diagnostic testing, it is imperative to optimize exercise MPI to increase its diagnostic yield and impact on management.

Cardiopulmonary exercise testing (CPET) is a noninvasive, objective assessment of the body's response to exercise and can evaluate additional components of the cardiovascular and pulmonary systems. This testing provides useful information on the functional status of patients with heart failure, chronic pulmonary disease, and patients with exercise intolerance. The AHA/ACC endorse the use of CPET in patients with heart failure to determine the etiology of exertional intolerance as well as for prognostication (class IIa recommendation). ${ }^{3}$ The American Thoracic Society/American College of Chest Physicians (ATS/ACCP) endorse the use of CPET for

Reprint requests: Lawrence M. Phillips, MD, Leon H. Charney Division of Cardiology, New York University Langone Medical Center, 530 First Ave, Skirball Sk-9U, New York, NY 10016; lawrence.phillips@nyumc.org

J Nucl Cardiol 2019;26:107-8.

1071-3581/\$34.00

Copyright (C) 2017 American Society of Nuclear Cardiology. evaluation of exercise intolerance, unexplained dyspnea, and evaluation and prognostication of known cardiac or pulmonary disease. ${ }^{4}$ Therefore, this raises two questions: should cardiologists be using CPET more frequently as part of a comprehensive diagnostic evaluation strategy? And could the addition of CPET optimize the diagnostic yield and impact on management of exercise MPI?

In this issue of the Journal, Christopoulos et al. report on a single center retrospective evaluation of the impact of a combined exercise MPI and CPET testing strategy on diagnosis and patient management. ${ }^{5}$ The study cohort was 415 patients with complaints of dyspnea $(51 \%)$, chest pain $(31 \%)$, pre-transplant or preoperative evaluation (9\%), arrhythmia-related syncope $(6 \%)$, or for risk stratification in asymptomatic patients (4\%). Gated perfusion SPECT imaging was evaluated for evidence of myocardial ischemia, infarction, and left ventricular ejection fraction (LVEF); while CPET performance was assessed for deconditioning, cardiovascular impairment, and pulmonary impairment. Data on subsequent patient management were collected via chart review and analyzed for changes in management of pulmonary disease, coronary disease, heart failure, or deconditioning. A significant finding was a difference in management of patients with normal exercise MPI and abnormal CPET results. These patients received significantly more subsequent pulmonary work-ups $(37.2 \%$ vs $4.0 \%, p<0.001)$, more referrals for cardiac rehabilitation for deconditioning (33.5\% vs $17.4 \%, p=0.004)$, and more augmentation in medical therapy for heart failure $(20.0 \%$ vs $8.1 \%$, $p=0.006$ ). These findings suggest that for non-specific symptoms, alternative diagnoses beyond flow-limiting CAD should always be considered. As expected, CPET results after a normal exercise MPI did not alter the rate of coronary angiography referrals. In patients with abnormal exercise MPI results, the CPET result had 
little impact on subsequent management and no difference in management of pulmonary disease, heart failure, or deconditioning. These findings suggest that in most patients the addition of CPET was not beneficial; however, in select patients the results of the exercise MPI and CPET co-testing did impact management, especially when the results were divergent. We agree with the authors that a subgroup analysis would be useful to better define which patients benefited from upfront cotesting based on their pre-test probability, but the current analysis was likely underpowered which lead to no significant findings.

The findings in this study are important because they highlight the potential value of performing CPET in conjunction with exercise MPI in select patients and the impact this testing can have on management. However, there are three areas that will be important to address in future investigation. First, should the pre-test probability for all possible diagnoses be considered before testing? For example, if a patient has moderate pre-test probability for $\mathrm{CAD}$ and high pre-test probability for deconditioning, would the addition of CPET truly add diagnostic value to a normal exercise MPI? Perhaps ruling out $\mathrm{CAD}$ would be sufficient to make deconditioning the presumptive diagnosis. Similarly, if a patient has high pre-test probability for flow-limiting CAD and low pre-test probability for non-coronary disease, exercise MPI would not be the test of choice for coronary evaluation and CPET results would be unlikely to alter near-term management. This is supported by the findings in the present study where Christopoulos et al. did not detect a change in management of patients with positive exercise MPI results and differing CPET results. If we are to use combined exercise MPI and CPET testing to assess for multiple diagnoses in a single-test strategy, the right patient for this strategy should be clearly defined based on their pre-test probability for all the diagnoses under evaluation. Second, the present study had no control group that underwent a standard cardiovascular work-up. Therefore, we cannot say with certainty exactly how this upfront co-testing strategy changed patient management. We can only infer that the different results of the exercise MPI and CPET were associated with different downstream patient management. Finally, future investigation should report longterm patient outcomes to demonstrate if the addition of CPET, and the changes in management, improves patient outcomes. CPET is an important tool in our diagnostic armamentarium, but it is expensive, difficult to perform, and is often carried out only at specialized centers. In addition, as the results of the present study demonstrate, routine combined testing of all-comers would likely be unnecessary and excessive. Showing that combined testing actually improves patient outcomes would help validate this more expensive testing strategy and justify its expanded use in clinical practice.

The authors should be commended for a well-executed analysis of how upfront exercise MPI and CPET cotesting impacted the subsequent management of allcomers with various presenting symptoms and various pre-test probabilities for flow-limiting CAD. The present study demonstrates that combined exercise MPI and CPET testing appears to impact the clinician's decisionmaking and subsequent patient management, especially in patients who have normal exercise MPI studies. Patients with abnormal exercise MPI results derived less benefit from this one-test strategy, a signal that the right patient for this form of comprehensive work-up is the patient with a low pre-test probability for flow-limiting CAD. However, further investigation is needed to confirm their findings as well as determine if exercise MPI and CPET co-testing ultimately improves patient outcomes.

\section{Disclosures}

The authors have indicated that they have no financial conflict of interest.

\section{References}

1. Ladapo JA, Blecker S, Douglas PS. Physician decision making and trends in the use of cardiac stress testing in the United States: An analysis of repeated cross-sectional data. Ann Intern Med. 2014;161:482-90.

2. Romero J, Husain SA, Holmes AA, Kelesidis I, Chavez P, Mojadidi MK, et al. Non-invasive assessment of low risk acute chest pain in the emergency department: A comparative meta-analysis of prospective studies. Int J Cardiol. 2015;187:565-80.

3. Hunt SA, Abraham WT, Chin MH, Feldman AM, Francis GS, Ganiats TG, et al. ACC/AHA 2005 Guideline Update for the Diagnosis and Management of Chronic Heart Failure in the Adult: A report of the American College of Cardiology/American Heart Association Task Force on Practice Guidelines (Writing Committee to Update the 2001 Guidelines for the Evaluation and Management of Heart Failure): Developed in collaboration with the American College of Chest Physicians and the International Society for Heart and Lung Transplantation: endorsed by the Heart Rhythm Society. Circulation. 2005;112:e154-235.

4. American Thoracic S and American College of Chest P. ATS/ ACCP Statement on cardiopulmonary exercise testing. Am J Respir Crit Care Med. 2003;167:211-77.

5. Christopoulos et al. The impact of combined cardiopulmonary exercise testing and SPECT myocardial perfusion imaging on downstream evaluation and management. J Nuc Cardiol. 